\title{
Erratum: Diffractive deeply virtual Compton scattering [Phys. Rev. D 101, 074005 (2020)]
}

\author{
B. Pire®, L. Szymanowski, and S. Wallon
}

(Q) (Received 3 March 2021; published 22 March 2021)

DOI: 10.1103/PhysRevD.103.059901

A numerical mistake lead to an erroneous Fig. 3 that we correct here (Fig. 1). Moreover, a simplified formula relates the skewness variable to the virtuality of the final state virtual photon, and Eq. (11) can be changed to

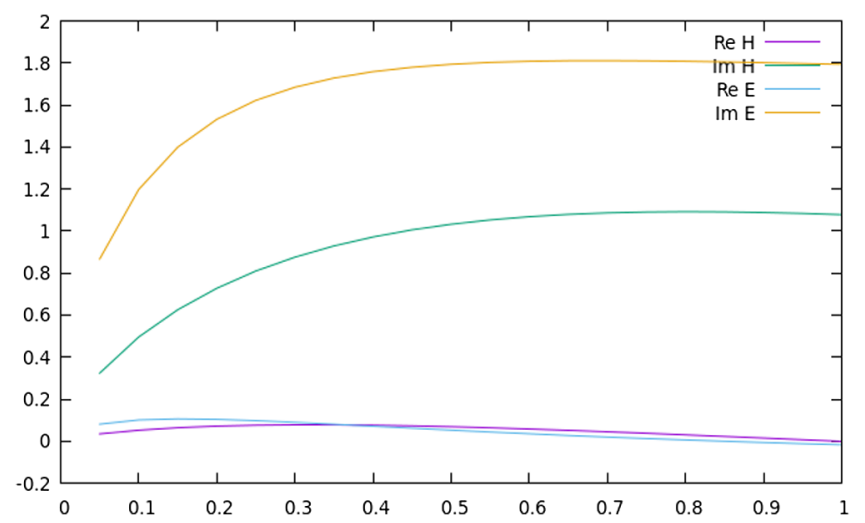

FIG. 1. (previous Fig. 3) An estimate of the real (lower curves) and imaginary (upper curves) parts of the diffractive Compton form factors $\mathcal{H}^{d}$ and $\mathcal{E}^{d}$ at $Q^{2}=Q^{\prime 2}=4 \mathrm{GeV}^{2}$ and $t=-0.1 \mathrm{GeV}^{2}$, divided by the prefactor $6 f_{\rho} K / \sqrt{2} Q^{4}$ from Eq. (23).

$$
\xi \approx \frac{Q^{\prime 2}}{2 s_{2}-Q^{\prime 2}}
$$

After completing a phenomenological study of the process [1], we finally should soften the sentence about the measurability of the process at future electron-ion colliders.

We thank Wim Cosyn for discovering our error and for helpful discussions.

[1] W. Cosyn and B. Pire, arXiv:2103.01411.

Published by the American Physical Society under the terms of the Creative Commons Attribution 4.0 International license. Further distribution of this work must maintain attribution to the author(s) and the published articles title, journal citation, and DOI. 